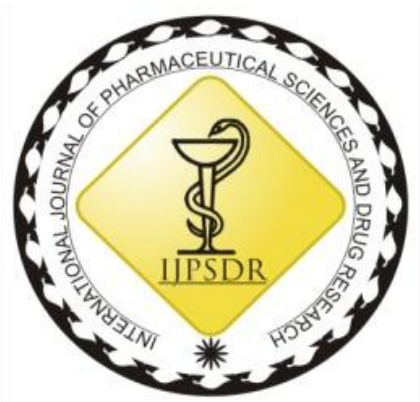

ISSN: 0975-248X

RESEARCH ARTICLE CODEN (USA): IJPSPP (cc) EY-NC-SA

\title{
Newly Developed Highly Sensitive Method for the Determination of Capecitabine by Using UV-Spectroscopy
}

\author{
Manas Ranjan Mishra*, Punam Agrawal, Surya Narayan Das \\ Gayatri College of Pharmacy, Sambalpur, Odisha-768200, India
}

Copyright (C) 2019 Manas Ranjan Mishra et al. This is an open access article distributed under the terms of the Creative Commons AttributionNonCommercial-ShareAlike 4.0 International License which allows others to remix, tweak, and build upon the work non-commercially, as long as the author is credited and the new creations are licensed under the identical terms.

\begin{abstract}
Capecitabine is a 'pro-drug' to the cytotoxic agent 5-fluorouracil (5-FU) intended to administered orally. Capecitabine is generally used as first line monotherapy for advanced colon cancer. Simple, rapid, accurate UV spectrophotometric methods were developed in the present study and validated for the estimation of Capecitabine in bulk and its formulations as per ICH guidelines. Three solvent systems viz., $0.1 \mathrm{~N} \mathrm{NaOH}, 0.1 \mathrm{~N} \mathrm{HCl}$ and $\mathrm{Methanol}$ : Water (1:3) was tried. The results suggest that the developed method shows linearity over the range of concentration $2-24 \mu \mathrm{g} / \mathrm{ml}$ and a correlation coefficient of 0.9999 . Accuracy, precision, linearity, robustness, and ruggedness were statistically validated as per ICH guidelines for all the developed methods. The \% RSD values for validated methods were found to be less than 1.5 and methods will find application in routine analysis of drug formulations containing Capecitabine.
\end{abstract}

Keywords: Capecitabine, UV-spectrophotometric, 0.1N NaOH, 0.1N HCl and Methanol: Water (1:3).

DOI: 10.25004/IJPSDR.2019.110304

Int. J. Pharm. Sci. Drug Res. 2019; 11(3): 91-97

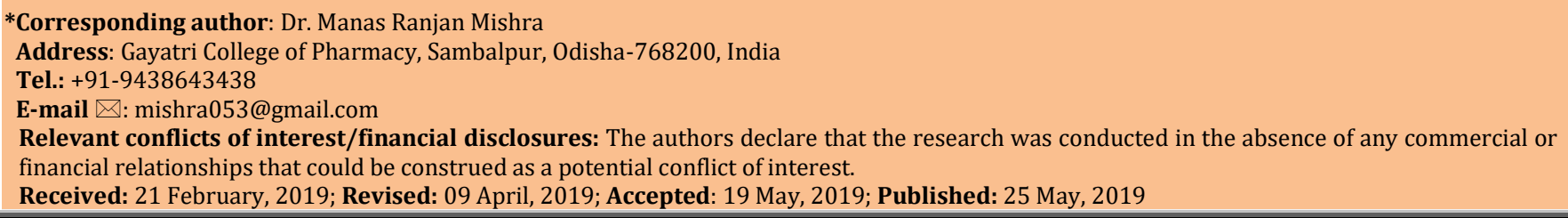

\section{INTRODUCTION}

The drug is official in the Indian Pharmacopoeia (IP). [12] Capecitabine $\left(\mathrm{C}_{15} \mathrm{H}_{22} \mathrm{FN}_{3} \mathrm{O}_{6}\right)$ is 5-deoxy-5-fluoro-N(pentyloxy) carbonyl]-cytidinewith molecular weight 359.35. [3-4] The synthesis of thymidine monophosphate inhibited by 5-FU, thymidine monophosphate is an active form of thymidine which is required for de novo synthesis of DeoxyRibo Nucleic acid (DNA).

Capecitabine is an orally administered chemotherapeutic agent used in the treatment of metastatic breast and colorectal cancers and is a prodrug of 5'-deoxy-5- fluorouridine (5'-DFUR), which is enzymatically converted to 5-fluorouracil in the tumor, where it inhibits DNA synthesis and slows growth of tumor tissue. The activation of Capecitabine follows a pathway with three enzymatic steps and two intermediary metabolites, 5'- deoxy-5-fluorocytidine (5'DFCR) and 5'-deoxy-5- fluorouridine (5'-DFUR), to form 5-fluorouracil. Chemically it is 5'-deoxy-5-fluoro$\mathrm{N}$-[(pentyloxy) carbonyl] - cytidine (figure 1) with empirical formula $\mathrm{C}_{15} \mathrm{H}_{22} \mathrm{FN}_{3} \mathrm{O}_{6}$ and the molecular weight of $359.35 \mathrm{~g} / \mathrm{mol}$. [5]

A very few methods appeared in the literature for the assay of Capecitabine in biological fluids and 
pharmaceutical formulations viz., HPLC [6-9], LC-UV [1011], LC-MS [12], and LMS/MS [13] methods. Hence in the present work it was aimed to develop and validate accurate, precise, simple and rapid UV spectroscopic methods for the estimation of Capecitabine in bulk and its formulations as per ICH guidelines.

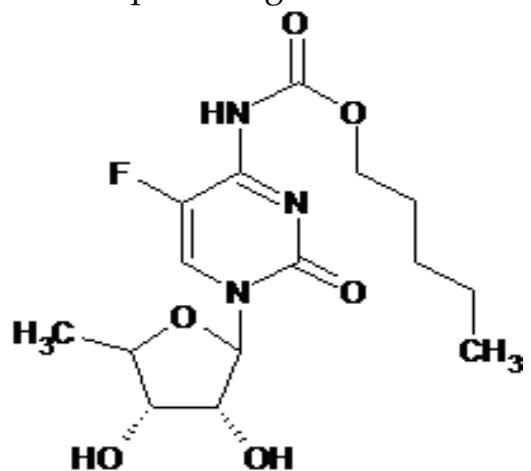

pentyl $\{1-[(3 R, 4 S)-3,4$-dihydroxy-5-methyltetrahydrofuran-2-yl]-5fluoro-2-oxo-1,2-dihydropyrimidin-4-yl\}carbamate

Fig. 1: Chemical structure of Capecitabine

\section{MATERIALS AND METHODS}

Capecitabine gift sample was obtained. Procured methanol, hydrochloric acid and sodium hydroxide from S.D Fine chemicals, Mumbai and double distilled water were used throughout the experiments. The other chemicals used were of analytical grade.

\section{METHODS}

Determination of absorption maxima ( $\lambda$ max)

Preparation of Capecitabine standard stock solution $(1000 \mu \mathrm{g} / \mathrm{ml})$

About $50 \mathrm{mg}$ of Capecitabine working standard weighed accurately and transferred to a $50 \mathrm{ml}$ volumetric flask. To it $40 \mathrm{ml}$ of $0.1 \mathrm{~N} \mathrm{NaOH}$ was added and was shaken for 5 minutes to dissolve and the volume was made up to $50 \mathrm{ml}$ with $0.1 \mathrm{~N} \mathrm{NaOH}$. Similarly a standard stock solution was prepared in $0.1 \mathrm{~N} \mathrm{HCl}$ and Methanol: Water (1:3) solvent systems.

Preparation of Capecitabine sample solution

Transferred aliquots of standard stock solution into a series of $10 \mathrm{ml}$ volumetric flask and diluted with $0.1 \mathrm{~N}$ $\mathrm{NaOH}$ to get desired concentrations. Similarly sample solutions were prepared from above stock solution. UV scanning was done for the sample solutions in the range of 200-380 $\mathrm{nm}$ using double beam UV Spectrophotometer and absorption maxima of Capecitabine was determined.

\section{Determination of linearity range}

Standard solutions of Capecitabine in the concentration range of $4-40 \mu \mathrm{g} / \mathrm{ml}$ were prepared in $0.1 \mathrm{~N} \mathrm{NaOH}$ and absorbance was measured at $292.8 \mathrm{~nm}$ taking the $0.1 \mathrm{~N}$ $\mathrm{NaOH}$ as the blank. Similarly absorbance of Capecitabine in the concentration range of $4-40 \mu \mathrm{g} / \mathrm{ml}$ in $0.1 \mathrm{~N} \mathrm{HCl}$, and Methanol: Water (1:3) were measured at $304 \mathrm{~nm}$ and $300.8 \mathrm{~nm}$ respectively using $0.1 \mathrm{~N} \mathrm{HCl}$, and Methanol: Water (1:3) solvent systems as blank.

\section{Calibration curve}

Appropriate aliquots from standard stock solutions of Capecitabine were transferred to series of $10 \mathrm{ml}$ volumetric flasks. The volume was adjusted to the mark with $0.1 \mathrm{~N} \mathrm{NaOH}$ to obtain concentrations of 4,8 , $12,16,20$ and $24 \mu \mathrm{g} / \mathrm{ml}$ and the absorbance was measured at $292.8 \mathrm{~nm}$. Similarly a set of same concentrations were prepared in $0.1 \mathrm{~N} \mathrm{HCl}$ and Methanol: Water (1:3). The absorbance was Measure at $304 \mathrm{~nm}$ and $300.8 \mathrm{~nm}$ respectively against respective solvent systems as blank. The concentration vs absorbance values were plotted and interpreted.

\section{Accuracy Validation}

The accuracy was evaluated by applying the proposed methods to the analysis formulations with known amounts of drug. The accuracy was calculated with respect to percentage of the drug recovered from the formulations.

\section{Capecitabine standard solution preparation (for bulk)} Accurately weighed $50 \mathrm{mg}$ of Capecitabine working standard was transferred to a $50 \mathrm{ml}$ volumetric flask, about $40 \mathrm{ml}$ of $0.1 \mathrm{~N} \mathrm{NaOH}$ was added to dissolve. Diluted up to the mark with $0.1 \mathrm{~N} \mathrm{NaOH}$ and mixed. To get desired concentrations, aliquots of stock solution were further diluted with $0.1 \mathrm{~N} \mathrm{NaOH}$. Similarly standard solutions were prepared in other solvent systems viz., $0.1 \mathrm{~N} \mathrm{HCl}$, Methanol: Water (1:3).

\section{Capecitabine sample preparation (for tablets)}

Accurately weighed 5 tablets were grounded in a mortar and transferred the equivalent to $50 \mathrm{mg}$ of Capecitabine into a $50 \mathrm{ml}$ volumetric flask. Added 40 $\mathrm{ml}$ of $0.1 \mathrm{~N} \mathrm{NaOH}$ and was shaken it for $1 \mathrm{~h}$. Diluted to volume with $0.1 \mathrm{~N} \mathrm{NaOH}$ and the contents was mixed and filtered through $0.45 \mu \mathrm{m}$ membrane filter. Aliquots of the filtrate were transferred to $25 \mathrm{ml}$ volumetric flask and diluted to volume with the $0.1 \mathrm{~N} \mathrm{NaOH}$ to got desired concentration. Similarly sample solutions were prepared in other solvent systems viz., $0.1 \mathrm{~N} \mathrm{HCl}$, Methanol: Water (1:3). Recovery studies were carried out by adding known amount of standard drug (40\% and $20 \%$ ) to the sample solution. The absorbance measured and the amount was calculated from the calibration curve. The recovery percentage was calculated in terms of RSD percentage which was less than $2 \%$.

\section{Precision}

The precision was determined by intraday and inter day observations. Repeatability was evaluated assaying 3 determinations at the same concentration $(10 \mu \mathrm{g} / \mathrm{ml})$, during the same day, under the same experimental conditions. Intermediate precision was analyzed comparing the assays in 3 determinations at the same concentration $(10 \mu \mathrm{g} / \mathrm{ml})$ during 3 different days. Precision (repeatability and intermediate precision) was expressed as relative standard deviation (RSD).

Sample preparation (for tablets)

Accurately weighed 5 tablets were grounded in a mortar and transferred equivalent to $50 \mathrm{mg}$ of Capecitabine into a $50 \mathrm{ml}$ volumetric flask, $40 \mathrm{ml}$ of $0.1 \mathrm{~N} \mathrm{NaOH}$ was added and shaken for $1 \mathrm{~h}$. Diluted to volume with $0.1 \mathrm{~N} \mathrm{NaOH}$. The contents mixed and were filtered through $0.45 \mu \mathrm{m}$ membrane filter. Aliquots of 
the filtrate was transferred to $25 \mathrm{ml}$ volumetric flask and diluted to volume with the $0.1 \mathrm{~N} \mathrm{NaOH}$ to got desired concentration. Similarly sample preparations were prepared in other solvent systems viz., $0.1 \mathrm{~N} \mathrm{HCl}$, Methanol: Water (1:3).

Intraday precision was determined by for three times in the same day analyzing Capecitabine (morning, afternoon, evening) at respective absorption maxima using respective solvent systems. Interday precision was determined by analyzing daily once (morning) for three days at respective absorption maxima using respective solvent systems. The percentage RSD values were calculated and it should be less than $2 \%$.

\section{LOD and LOQ}

LOD/LOQ parameters are always useful to demonstrate that the analysis is being conducted in a region which is above the LOQ value. The LOD and LOQ were calculated based on the standard deviation of the response ( $y$ intercepts of regression lines) and the slope using three independent analytical curves, as denied by ICH.

$$
\begin{aligned}
& \operatorname{LOD}(\mu \mathrm{g} / \mathrm{ml})=3.3 \times \frac{\mathrm{\sigma}}{\mathrm{s}} \\
& \operatorname{LOQ}(\mu \mathrm{g} / \mathrm{ml})=10 \times \underline{\sigma}
\end{aligned}
$$

Where $\sigma$ - Standard deviation of the response: s - Slope ratio curve.

The lowest possible concentration where the drug Capecitabine shows response was determined in all the solvent systems. The absorbance at this concentration was measured in triplicate in respective solvent systems at respective absorption maxima. The LOD/LOQ was calculated by using formulae from the data obtained.

\section{Robustness}

Robustness of the proposed methods was determined by the analysis of samples and standard solutions $(10 \mu \mathrm{g} / \mathrm{ml})$ at different wavelengths $( \pm 5 \mathrm{~nm})$ and at different solution temperatures (refrigeration condition $2-8^{\circ} \mathrm{C}$ and $37^{\circ} \mathrm{C}$ ). The stability study was performed maintaining the drug working solution in respective solvent systems for $48 \mathrm{~h}$ protected from light, looking for the decrease of absorbance compared with those of freshly prepared solutions to assess the stability of drug.

Appropriate concentrations of Capecitabine from bulk and formulations were prepared in respective solvent systems. Analysis was carried out at three different wavelengths (actual and $\pm 5 \mathrm{~nm}$ ). Amount found was calculated at three different wavelengths in terms of percentage RSD and values were less than $2 \%$.

\section{Ruggedness}

Ruggedness is not addressed in the ICH documents; it is a measure of reproducibility of test results under normal, expected operational conditions from analyst to analyst and instrument to instrument.

Appropriate concentrations of Capecitabine from bulk and formulations were prepared in respective solvent systems. Analysis was carried out by two different analysts and also two instruments. Amount found was calculated at three different wavelengths in terms of percentage RSD and values were less than $2 \%$.

Table 1: Linearity range curve of Capecitabine in $0.1 \mathrm{~N} \mathrm{NaOH}, 0.1 \mathrm{~N}$

\begin{tabular}{|c|c|c|c|}
\hline \multirow{2}{*}{$\begin{array}{l}\text { Conc. } \\
(\mu \mathrm{g} / \mathrm{ml})\end{array}$} & $0.1 \mathrm{~N} \mathrm{NaOH}$ & $0.1 \mathrm{~N} \mathrm{HCl}$ & $\begin{array}{c}\text { Methanol: Water } \\
(1: 3)\end{array}$ \\
\hline & $\begin{array}{c}\text { Absorbance }^{*} \pm \\
\text { SD }\end{array}$ & $\begin{array}{c}\text { Absorbance* } \pm \\
\text { SD }\end{array}$ & Absorbance $^{*} \pm$ SD \\
\hline 4 & $0.0672 \pm 0.0004$ & $0.0677 \pm 0.0003$ & $0.0670 \pm 0.003$ \\
\hline 8 & $0.118 \pm 0.0021$ & $0.123 \pm 0.0021$ & $0.124 \pm 0.0022$ \\
\hline 12 & $0.178 \pm 0.0016$ & $0.183 \pm 0.0016$ & $0.188 \pm 0.0016$ \\
\hline 16 & $0.237 \pm 0.0010$ & $0.242 \pm 0.0010$ & $0.243 \pm 0.0011$ \\
\hline 20 & $0.302 \pm 0.0049$ & $0.307 \pm 0.0049$ & $0.308 \pm 0.0048$ \\
\hline 24 & $0.362 \pm 0.0020$ & $0.368 \pm 0.0020$ & $0.369 \pm 0.0021$ \\
\hline 28 & $0.382 \pm 0.0011$ & $0.421 \pm 0.0011$ & $0.387 \pm 0.0011$ \\
\hline 32 & $0.412 \pm 0.0032$ & $0.441 \pm 0.0032$ & $0.412 \pm 0.0032$ \\
\hline 36 & $0.472 \pm 0.0023$ & $0.473 \pm 0.0023$ & $0.462 \pm 0.0023$ \\
\hline 40 & $0.512 \pm 0.0033$ & $0.511 \pm 0.0033$ & $0.489 \pm 0.0023$ \\
\hline
\end{tabular}
$\mathrm{HCl}$ and Methanol: Water (1:3) solvent systems.

Table 2: Calibration curve data of Capecitabine in $0.1 \mathrm{~N} \mathrm{NaOH}, 0.1 \mathrm{~N}$ $\mathrm{HCl}$ and Methanol: Water (1:3) solvent systems served absorption maxima.

\begin{tabular}{cccc}
\hline \multirow{2}{*}{$\begin{array}{c}\text { Conc. } \\
(\mu \mathrm{g} / \mathbf{m l})\end{array}$} & $\mathbf{0 . 1} \mathbf{~ N ~ N a O H}$ & $\mathbf{0 . 1} \mathbf{~ N C l}$ & $\begin{array}{c}\text { Methanol: Water } \\
(\mathbf{1}: 3)\end{array}$ \\
\cline { 2 - 4 } & $\begin{array}{c}\text { Absorbance* } \\
\text { SD }\end{array}$ & $\begin{array}{c}\text { Absorbance } \\
\text { SD }\end{array}$ & Absorbance \pm SD \\
\hline 0 & $0.0000 \pm 0.0000$ & $0.0000 \pm 0.0000$ & $0.0000 \pm 0.0000$ \\
4 & $0.0622 \pm 0.0012$ & $0.0658 \pm 0.0015$ & $0.0637 \pm 0.0018$ \\
8 & $0.1180 \pm 0.0015$ & $0.1240 \pm 0.0019$ & $0.1230 \pm 0.0026$ \\
12 & $0.1780 \pm 0.0019$ & $0.1840 \pm 0.0021$ & $0.1830 \pm 0.0029$ \\
16 & $0.2370 \pm 0.0021$ & $0.2470 \pm 0.0024$ & $0.2460 \pm 0.0032$ \\
20 & $0.2970 \pm 0.0026$ & $0.3080 \pm 0.0026$ & $0.3070 \pm 0.0023$ \\
24 & $0.3560 \pm 0.0018$ & $0.3690 \pm 0.0021$ & $0.3680 \pm 0.0019$ \\
\hline
\end{tabular}

Table 3: Statistical data of calibration curve for Capecitabine in $0.1 \mathrm{~N} \mathrm{NaOH}, 0.1 \mathrm{~N} \mathrm{HCl}$ and Methanol: Water (1:3) solvent systems.

\begin{tabular}{cccc}
\hline Parameters & $\mathbf{0 . 1} \mathbf{~ N ~ N a H}$ & $\mathbf{0 . 1} \mathbf{~ N C l}$ & $\begin{array}{c}\text { Methanol: } \\
\text { Water (1:3) }\end{array}$ \\
\hline $\begin{array}{c}\text { Mmax }(\mathrm{nm}) \\
\text { Beer's range } \\
(\mu \mathrm{g} / \mathrm{ml})\end{array}$ & 292.8 & 304.0 & 300.8 \\
Molar & $24 \mu \mathrm{g} / \mathrm{ml}$ & $2-24 \mu \mathrm{g} / \mathrm{ml}$ & $2-24 \mu \mathrm{g} / \mathrm{ml}$ \\
absorptivity & $1.714 \times 104$ & $1.716 \times 104$ & $1.711 \times 104$ \\
(mol-1cm1) & & & \\
Best fit valves & & & \\
Slope & $0.0147 \pm$ & $0.0153 \pm$ & $0.01531 \pm$ \\
Y-intercept & 0.000057 & 0.000050 & 0.000076 \\
when X=0.0 & $0.00082 \pm$ & $0.00078 \pm$ & $0.001714 \pm$ \\
X-intercept & 0.00082 & 0.00072 & 0.0011 \\
when Y=0.0 & -0.05554 & -0.05135 & -0.1120 \\
1/Slope & 67.61 & 65.36 & 65.33 \\
95\% CI & & & 0.01511 to \\
Slope & 0.01464 to & 0.01517 to & 0.01550 \\
r2 & 0.01494 & 0.01543 & $<0.0001$ \\
P valve & 0.9999 & 0.9999 & \\
& $<0.0001$ & $<0.0001$ & $<99$ \\
\hline
\end{tabular}

\section{RESULTS AND DISCUSSION}

Simple, rapid, economic, accurate, precise and sensitive UV spectrophotometric methods were developed and validated as per ICH guideline and USP 2000 for the estimation of Capecitabine in bulk and formulations. Three different solvent systems viz., $0.1 \mathrm{~N} \mathrm{NaOH}, 0.1 \mathrm{~N}$ $\mathrm{HCl}$ and Methanol: Water (1:3) was selected. The developed methods were further validated for 
accuracy, precision, LOD, LOQ, specificity, robustness and ruggedness with statistical data.

Table 4: Data showing accuracy of Capecitabine (bulk) in all solvent systems.

\begin{tabular}{|c|c|c|c|}
\hline \multicolumn{4}{|c|}{ Method $10.1 \mathrm{~N} \mathrm{NaOH}$} \\
\hline \multirow[t]{2}{*}{ Sample No } & \multicolumn{2}{|c|}{$\begin{array}{l}\text { Concentration of Capecitabine } \\
\qquad(\mu \mathrm{g} / \mathrm{ml})\end{array}$} & \multirow{2}{*}{$\begin{array}{c}\% \text { of } \\
\text { recovery }\end{array}$} \\
\hline & Theoritical & Experimentaly & \\
\hline 1 & 2 & 2 & 100 \\
\hline 2 & 4 & 3.9 & 98.7 \\
\hline 3 & 6 & 6 & 100 \\
\hline 4 & 8 & 8.04 & 100.5 \\
\hline 5 & 10 & 9.97 & 99.7 \\
\hline \multicolumn{4}{|c|}{ Method II 0.1 N HCl } \\
\hline & Theoritical & Experimentaly & \\
\hline 1 & 2 & 2.01 & 100.5 \\
\hline 2 & 4 & 4.02 & 100.5 \\
\hline 3 & 6 & 5.9 & 99.2 \\
\hline 4 & 8 & 8.0 & 100 \\
\hline 5 & 10 & 10.2 & 100.2 \\
\hline \multicolumn{4}{|c|}{ Method III Methanol : Water (1:3) } \\
\hline & Theoritical & Experimentaly & \\
\hline 1 & 2 & 2.02 & 101.1 \\
\hline 2 & 4 & 3.9 & 99.4 \\
\hline 3 & 6 & 6.03 & 100.5 \\
\hline 4 & 8 & 8.04 & 100.5 \\
\hline 5 & 10 & 10.06 & 100.6 \\
\hline
\end{tabular}

Table 5: Data showing recovery studies of capecitabine (formulations) in all solvent systems.

\begin{tabular}{|c|c|c|c|c|c|}
\hline \multirow{2}{*}{$\begin{array}{c}\text { Amount } \\
\text { present } \\
\text { in formulation } \\
(\mu \mathrm{g} / \mathrm{ml})\end{array}$} & \multicolumn{2}{|c|}{$\begin{array}{c}\text { Amount } \\
\text { added }\end{array}$} & \multirow{2}{*}{$\begin{array}{c}\text { Amount } \\
\text { recovered } \\
(\mu \mathrm{g} / \mathrm{ml})\end{array}$} & \multirow{2}{*}{$\begin{array}{l}\text { Mean \% } \\
\text { Recovery }\end{array}$} & \multirow{2}{*}{ RSD } \\
\hline & $\mu \mathrm{g}$ & $\%$ & & & \\
\hline \multirow[b]{2}{*}{10} & 4 & 40 & 13.9 & $99.2 \pm 1.909$ & 1.924 \\
\hline & 2 & 20 & 11.8 & $98.7 \pm 1.779$ & 1.802 \\
\hline \multirow{2}{*}{$\begin{array}{c}\text { Amount } \\
\text { present } \\
\text { in formulation } \\
(\mu \mathrm{g} / \mathrm{ml})\end{array}$} & \multicolumn{2}{|c|}{$\begin{array}{c}\text { Amount } \\
\text { added }\end{array}$} & \multirow{2}{*}{$\begin{array}{c}\text { Amount } \\
\text { recovered } \\
(\mu \mathrm{g} / \mathrm{ml}\end{array}$} & \multirow[t]{2}{*}{$\begin{array}{l}\text { Mean \% } \\
\text { Recovery }\end{array}$} & \multirow{2}{*}{ RSD } \\
\hline & $\mu \mathrm{g}$ & $\%$ & & & \\
\hline \multirow{2}{*}{10} & 4 & 40 & 14.03 & $100.2 \pm 1.808$ & 1.824 \\
\hline & 2 & 20 & 12.03 & $100.3 \pm 2.219$ & 2.102 \\
\hline \multirow{2}{*}{$\begin{array}{c}\text { Amount } \\
\text { present } \\
\text { in formulation } \\
(\mu \mathrm{g} / \mathrm{ml})\end{array}$} & \multicolumn{2}{|c|}{$\begin{array}{c}\text { Amount } \\
\text { added }\end{array}$} & \multirow{2}{*}{$\begin{array}{c}\text { Amount } \\
\text { recovered } \\
(\mu \mathrm{g} / \mathrm{ml}\end{array}$} & \multirow[t]{2}{*}{$\begin{array}{l}\text { Mean \% } \\
\text { Recovery }\end{array}$} & \multirow{2}{*}{ RSD } \\
\hline & $\mu \mathrm{g}$ & $\%$ & & & \\
\hline \multirow{2}{*}{10} & 4 & 40 & 14.1 & $100.9 \pm 2.707$ & 1.691 \\
\hline & 2 & 20 & 12.1 & $101.1 \pm 3.143$ & 1.130 \\
\hline
\end{tabular}

Table 6: Data showing LOD/LOQ of Capecitabine in all solvent systems.

\begin{tabular}{|c|c|c|}
\hline \multicolumn{3}{|c|}{ Method I 0.1 N NaOH } \\
\hline & Mean \pm SD & SEM \\
\hline Limit of detection & $0.191 \pm 0.046$ & 0.026 \\
\hline Limit of quantitation & $0.584 \pm 0.145$ & 0.084 \\
\hline \multicolumn{3}{|c|}{ Method II 0.1 N HCl } \\
\hline & Mean \pm SD & SEM \\
\hline Limit of detection & $0.48 \pm 0.047$ & 0.027 \\
\hline Limit of quantitation & $1.46 \pm 0.148$ & 0.084 \\
\hline \multicolumn{3}{|c|}{ Method III Methanol : Water (1:3) } \\
\hline & Mean \pm SD & SEM \\
\hline Limit of detection & $0.51 \pm 0.134$ & 0.072 \\
\hline Limit of quantitation & $1.54 \pm 0.384$ & 0.224 \\
\hline
\end{tabular}

The absorption maxima $(\lambda \max )$ with characteristic peak for capecitabine were found at $292.8 \mathrm{~nm}, 304.0 \mathrm{~nm}$ and $300.8 \mathrm{~nm}$ for $0.1 \mathrm{~N} \mathrm{NaOH}, 0.1 \mathrm{~N} \mathrm{HCl}$ and Methanol: Water (1:3) respectively. These absorption maxima were used to determine the linearity and it was shown linear relationship with correlation coefficient of 0.9999; 0.9999 and 0.9999 for $0.1 \mathrm{~N} \mathrm{NaOH}, 0.1 \mathrm{~N} \mathrm{HCl}$ and Methanol: Water (1:3) respectively in the concentration range of $2-24 \mu \mathrm{g} / \mathrm{ml}$. The spectra and data were shown in figure 2,3 and table 1 .
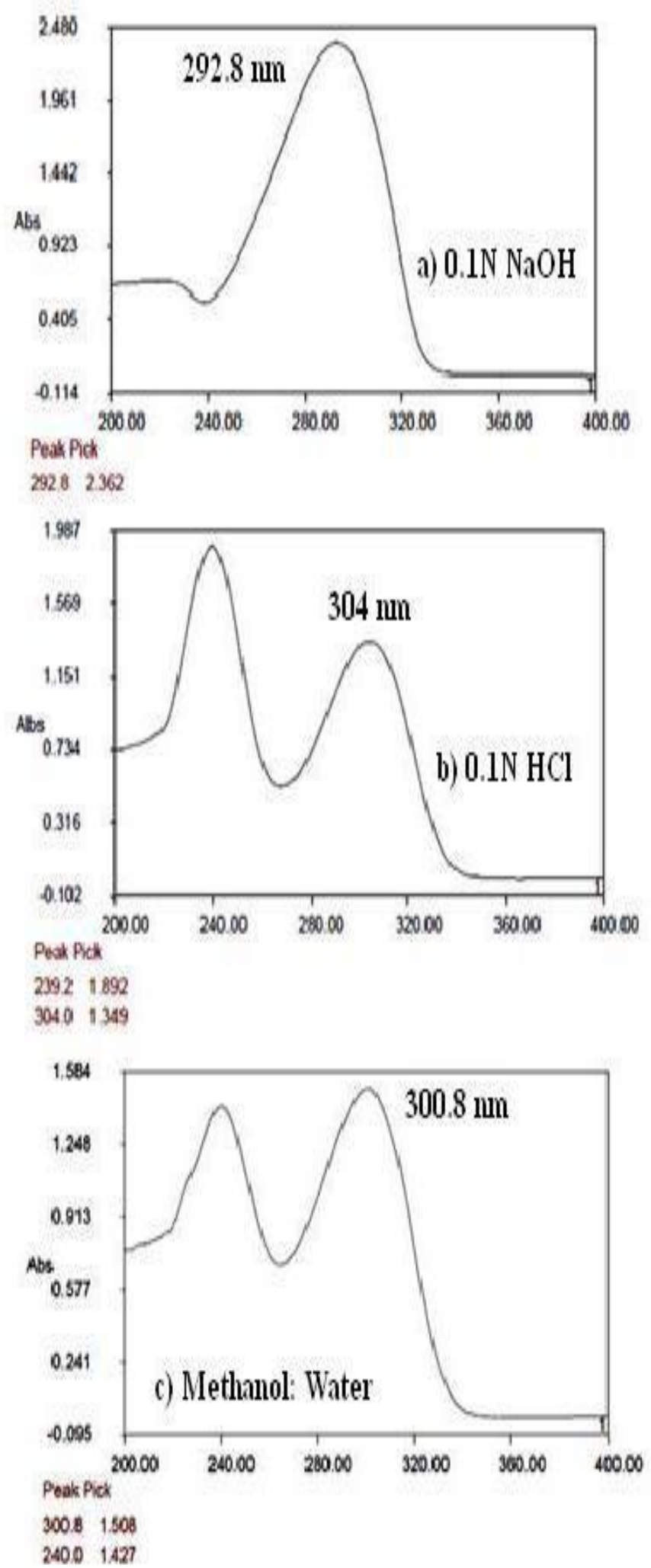

Fig. 2: Absorption maxima of Capecitabine in $0.1 \mathrm{~N} \mathrm{NaOH}, 0.1 \mathrm{~N}$ $\mathrm{HCl}$ and Methanol: Water (1:3) solvent systems. 

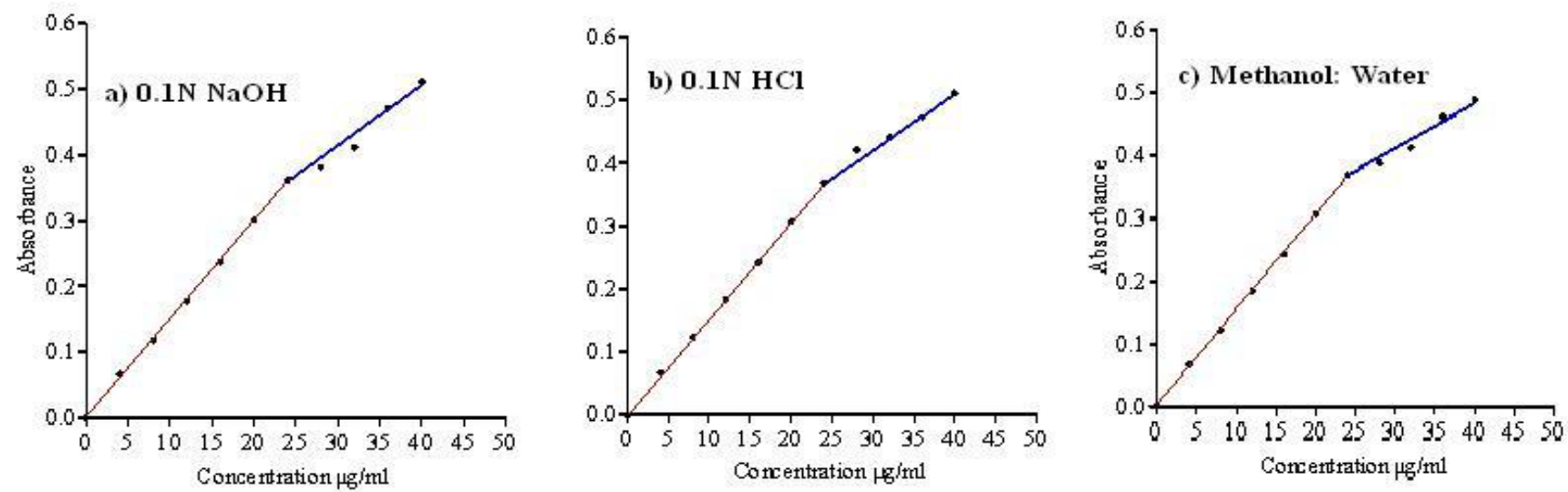

Fig. 3: Linearity range of Capecitabine in $0.1 \mathrm{~N} \mathrm{NaOH}, 0.1 \mathrm{~N} \mathrm{HCl}$ and Methanol: Water (1:3) solvent systems
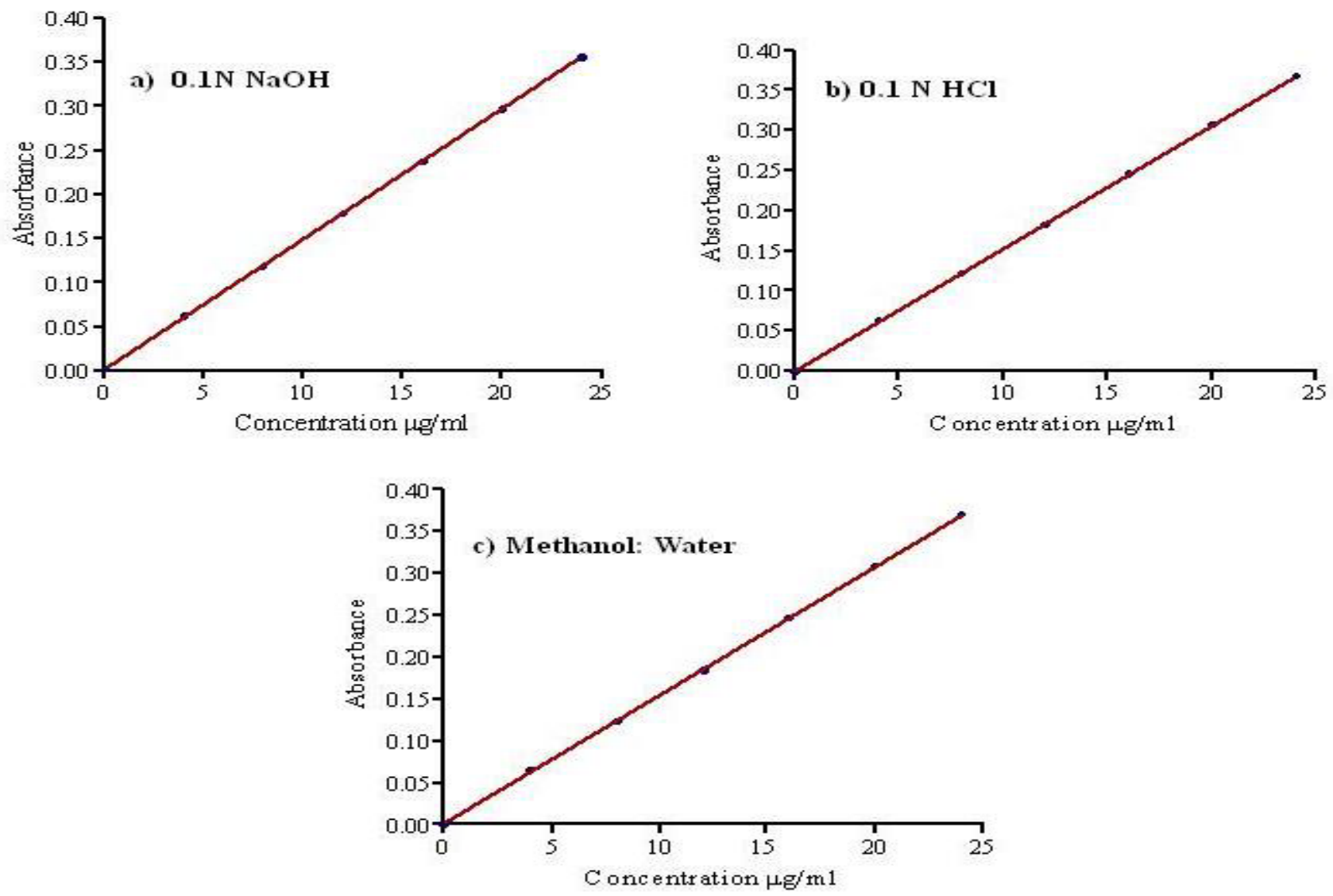

Fig. 4: Calibration curve of Capecitabine in $0.1 \mathrm{~N} \mathrm{NaOH}, 0.1 \mathrm{~N} \mathrm{HCl}$ and Methanol: Water (1:3) solvent systems.

Table 7: Data showing precision Intraday and Inter day trials with RSD values for Capecitabine in all solvent systems.

\begin{tabular}{|c|c|c|c|c|c|c|}
\hline \multicolumn{7}{|c|}{ Method I 0.1 N NaOH } \\
\hline Trials & Label claimed (mg/tab) & Amount found ( $\mathrm{mg} / \mathrm{tab})$ & $\%$ Label claime & $n \pm S D)$ & SEM & RSD \\
\hline \multirow{4}{*}{ Day-1 } & & 50.3 & $100.6 \pm 1.246$ & & 0.729 & 1.239 \\
\hline & 50 & 50.2 & $100.4 \pm 0.853$ & & 0.429 & 0.843 \\
\hline & & 49.5 & $99.0 \pm 0.642$ & & 0.373 & 0.654 \\
\hline & & Intra & $100.2 \pm 1.474$ & Intra & 0.851 & 1.473 \\
\hline \multirow[t]{3}{*}{ Day-2 } & 50 & 49.2 & $98.4 \pm 1.060$ & day & 0.611 & 1.062 \\
\hline & & trials & $99.2 \pm 1.385$ & trials & 0.860 & 1.388 \\
\hline & & 50.5 & $101.0 \pm 0.692$ & & 0.400 & 0.686 \\
\hline \multirow[t]{2}{*}{ Day-3 } & 50 & 50.01 & $100.02 \pm 0.961$ & & 0.200 & 0.959 \\
\hline & & 49.7 & $99.4 \pm 0.473$ & & 0.272 & 0.473 \\
\hline \multicolumn{7}{|c|}{ Method II 0.1 N HCl } \\
\hline \multirow{4}{*}{ Day-1 } & & 50.4 & $100.8 \pm 1.381$ & & 0.800 & 1.378 \\
\hline & 50 & 50.3 & $100.6 \pm 0.765$ & & 0.441 & 0.758 \\
\hline & & 50.2 & $100.4 \pm 1.024$ & Intra & 0.592 & 1.016 \\
\hline & & day & $100.8 \pm 0.662$ & day & 0.384 & 0.658 \\
\hline \multirow[t]{2}{*}{ Day-2 } & 50 & 50.2 & $100.4 \pm 1.204$ & & 0.693 & 1.196 \\
\hline & & 50.3 & $100.6 \pm 0.670$ & & 0.393 & 0.678 \\
\hline
\end{tabular}


Manas Ranjan Mishra et al. / Newly Developed Highly Sensitive Method for the Determination of Capecita..

\begin{tabular}{|c|c|c|c|c|c|c|c|}
\hline \multirow{3}{*}{ Day-3 } & \multirow{3}{*}{50} & 50.2 & \multicolumn{3}{|c|}{$100.4 \pm 0.201$} & \multirow{2}{*}{$\begin{array}{l}0.155 \\
0.860\end{array}$} & \multirow{2}{*}{$\begin{array}{l}0.199 \\
1.388\end{array}$} \\
\hline & & 49.9 & & $99.8 \pm 1.386$ & & & \\
\hline & & 49.6 & & $99.2 \pm 0.871$ & & 0.504 & 0.875 \\
\hline \multicolumn{8}{|c|}{ Method III Methanol : Water (1:3) } \\
\hline \multirow{4}{*}{ Day-1 } & & 49.9 & & $99.8 \pm 0.722$ & \multirow{9}{*}{$\begin{array}{l}\text { Intra } \\
\text { day } \\
\text { trials }\end{array}$} & 0.416 & 0.718 \\
\hline & 50 & 49.7 & & $99.4 \pm 0.791$ & & 0.458 & 0.796 \\
\hline & & 49.9 & & $99.8 \pm 0.792$ & & 0.458 & 0.803 \\
\hline & \multirow{3}{*}{50} & 50.6 & Intra & $101.2 \pm 1.155$ & & 0.665 & 1.156 \\
\hline \multirow[t]{2}{*}{ Day-2 } & & 50.2 & day & $100.4 \pm 1.363$ & & 0.913 & 1.575 \\
\hline & & 49.8 & trials & $99.6 \pm 0.757$ & & 0.437 & 0.760 \\
\hline \multirow{3}{*}{ Day-3 } & \multirow{3}{*}{50} & 50.2 & & $100.4 \pm 1.22$ & & 0.705 & 1.213 \\
\hline & & 49.8 & & $99.6 \pm 0.756$ & & 0.437 & 0.754 \\
\hline & & 49.7 & & $99.4 \pm 1.151$ & & 0.664 & 1.156 \\
\hline
\end{tabular}

Table 8: Data showing robustness of Capecitabine at different wavelengths in all solvent systems.

\begin{tabular}{|c|c|c|c|c|c|c|}
\hline Method & Conc. $(\mu \mathrm{g} / \mathrm{ml})$ & Wave length & Amount found & Mean $(\% \pm$ SD) & SEM & RSD \\
\hline \multirow{4}{*}{ Method I } & & 292 & 9.95 & $99.5 \pm 0.962$ & 0.552 & 0.905 \\
\hline & 10 & 297 & 8.67 & $86.7 \pm 1.735$ & 1.012 & 1.817 \\
\hline & & 287 & 8.7 & $87.0 \pm 1.013$ & 0.601 & 1.163 \\
\hline & & 304 & 9.93 & $99.3 \pm 0.712$ & 0.407 & 0.732 \\
\hline \multirow[t]{3}{*}{ Method II } & 10 & 309 & 8.7 & $87.0 \pm 1.507$ & 0.928 & 1.720 \\
\hline & & 299 & 8.8 & $88.0 \pm 1.059$ & 0.665 & 1.241 \\
\hline & & 300 & 9.98 & $99.8 \pm 1.241$ & 0.721 & 1.256 \\
\hline \multirow{2}{*}{ Method III } & 10 & 305 & 8.34 & $83.4 \pm 1.122$ & 0.702 & 1.332 \\
\hline & & 295 & 8.41 & $84.1 \pm 0.941$ & 0.562 & 1.076 \\
\hline
\end{tabular}

Table 9: Data showing ruggedness of Capecitabine by different Analysts in all solvent systems..

\begin{tabular}{|c|c|c|c|c|c|c|}
\hline Method & Conc. $(\mu \mathrm{g} / \mathrm{ml})$ & Analyst & Amount found & Recovery \pm SD & SEM & RSD \\
\hline \multirow{2}{*}{ Method I } & \multirow{2}{*}{10} & Analyst 1 & 10.01 & $100.1 \pm 0.54$ & 0.223 & 0.539 \\
\hline & & Analyst 2 & 9.94 & $99.4 \pm 0.3$ & 0.122 & 0.301 \\
\hline \multirow{2}{*}{ Method II } & \multirow{2}{*}{10} & Analyst 1 & 9.99 & $99.9 \pm 0.75$ & 0.307 & 0.756 \\
\hline & & Analyst 2 & 10.05 & $100.5 \pm 0.565$ & 0.231 & 0.565 \\
\hline \multirow{2}{*}{ Method III } & \multirow{2}{*}{10} & Analyst 1 & 10.01 & $100.1 \pm 0.41$ & 0.169 & 0.413 \\
\hline & & Analyst 2 & 9.99 & $99.9 \pm 0.393$ & 0.161 & 0.612 \\
\hline
\end{tabular}

Table 10: Data showing ruggedness of Capecitabine by using different Instruments in all solvent systems

\begin{tabular}{|c|c|c|c|c|c|c|}
\hline Method & Conc. $\mu \mathrm{g} / \mathrm{ml}$ ) & Instrument & Amount found & Recovery ( \pm SD) & SEM & RSD \\
\hline \multirow{2}{*}{ Method I } & \multirow{2}{*}{10} & Instrument 1 & 9.92 & $99.2 \pm 0.963$ & 0.554 & 0.964 \\
\hline & & Instrument 2 & 10.01 & $100.1 \pm 0.493$ & 0.284 & 0.492 \\
\hline \multirow{2}{*}{ Method II } & \multirow{2}{*}{10} & Instrument 1 & 9.7 & $97.0 \pm 0.709$ & 0.409 & 0.733 \\
\hline & & Instrument 2 & 9.71 & $97.1 \pm 0.642$ & 0.371 & 0.658 \\
\hline \multirow{2}{*}{ Method III } & \multirow{2}{*}{10} & Instrument 1 & 9.92 & $99.2 \pm 1.249$ & 0.721 & 1.251 \\
\hline & & Instrument 2 & 9.89 & $98.9 \pm 0.600$ & 0.346 & 0.610 \\
\hline
\end{tabular}

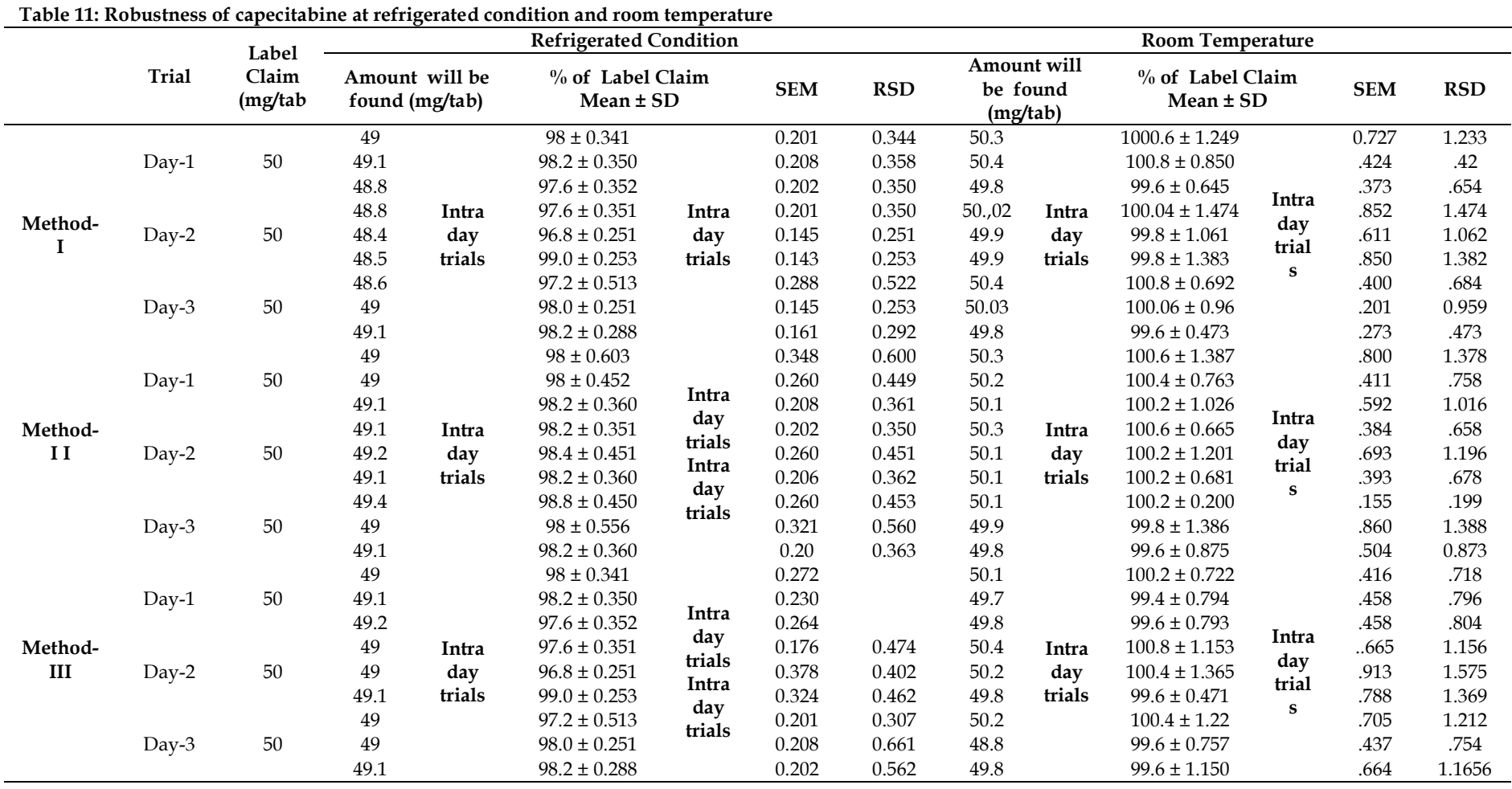


The proposed UV spectrophotometric methods were found to be simple, rapid, accurate, precise and economic. From the above data it was observed that all validation parameters met the predetermined acceptance criteria and validated in terms of linearity, accuracy, precision, reproducibility, robustness, and ruggedness as per the ICH guidelines. Thus it has been concluded that the proposed methods were validated for the analysis of capecitabine in bulk and its formulations.

\section{REFERENCES}

1. Brunton LL, Lazo JS, Parker KL. Goodman and Gilman: The pharmacological basis of therapeutics. Edn. 11, McGraw Hill Medical Publishing Division, 2006, pp. 1404-1405.

2. Hirsch BR, Zafar SY. Capecitabine in the management of colorectal cancer. Cancer Manag Res. 2011; 3: 79-89.

3. Walko CM, Lindley C. Capecitabine: a review. Clin Ther. 2005; 27: 23-44.

4. Milano G, Schellens JHM. Pyrimidine antimetabolites. In: Schellens JHM, McLeod HL, Newell DR. eds. Cancer Clinical Pharmacology. Oxford: University Press, 2005; pp. 51-62.

5. Indian Pharmacopoeia, 2010, 2, pp. 972-973.

6. Guichard SM, Mayer I, Jodrell DI. Simultaneous determination of capecitabine and its metabolites by HPLC and mass spectrometry for preclinical and clinical studies. J Chromatogr B. 2005; 826: 232-237.
7. Dhananjeyan MR, Liu J, Bykowski C, Trendel JA, Sarver JG, Andob $\mathrm{H}$, et al. Rapid and simultaneous determination of capecitabine and its metabolites in mouse plasma, mouse serum, and in rabbit bile by high-performance liquid chromatography. J Chromatogr A. 2007; 1138(1-2): 101-108.

8. Farkouh A, Ettlinger D, Schueller J, Georgopoulos A, Scheithauer W, Czejka M. A rapid and simple HPLC assay for quantification of capecitabine for drug monitoring purposes. Anticancer Res. 2010; 30: 5207-5212.

9. Piorkowska E, Kaza M, Fitatiuk J, Szlaska I, Pawinski T, Rudzki PJ. Rapid and simplified HPLCUV method with online wavelengths switching for determination of capecitabine in human plasma. Pharmazie. 2014; 69: 500-505.

10. Reigner B, Blesch K, Weidekamm E. Clinical pharmacokinetics of capecitabine. Springer Science+, Clin Pharmacokinet. 2001; 40: 85-104.

11. Zufia L, Aldaz A, Giraldez J. Simple determination of capecitabine and its metabolites by liquid chromatography with ultraviolet detection in a single injection. J Chromatogr B. 2004; 809: 51-58.

12. $\mathrm{Xu} \mathrm{Y,} \mathrm{Grem} \mathrm{JL.} \mathrm{Liquid} \mathrm{chromatography-mass} \mathrm{spectrometry}$ method for the analysis of the anticancer agent capecitabine and its nucleoside metabolites in human plasma. J Chromatogr B. 2003; 783: 273-285.

13. Siethoff C, Orth M, Ortling A, Brendel E, Redeker WW. Simultaneous determination of capecitabine and its metabolite 5-fluorouracil by column switching and liquid chromatographic/tandem mass spectrometry. J Mass Spect. 2004; 39: 884-889.

HOW TO CITE THIS ARTICLE: Mishra MR, Agrawal P, Das SN. Newly Developed Highly Sensitive Method for the Determination of Capecitabine by Using UV-Spectroscopy. Int. J. Pharm. Sci. Drug Res. 2019; 11(3): 91-97. DOI: 10.25004/IJPSDR.2019.110304 